\title{
CONVERGENCE OF MONOTONE DYNAMICAL SYSTEMS WITH MINIMAL EQUILIBRIA
}

\author{
JIANHONG WU
}

(Communicated by Kenneth R. Meyer)

\begin{abstract}
We show that each precompact orbit of strongly monotone dynamical systems on a Banach lattice $X$ is convergent if there is a continuous map $e: X \rightarrow E$, the set of equilibria, such that $e(x)$ is the maximal element in $E$ with $e(x) \leq x$. This result can be applied to study the convergence of a class of functional differential equations and partial differential equations.
\end{abstract}

Monotone dynamical systems have a strong tendency to converge to the set of equilibria. Under some compactness assumptions, Hirsch [3] shows that generically positive semiorbits are quasi-convergent, i.e. asymptotic to the set $E$ of equilibria. Therefore, when the set of equilibrium points has no accumulation point, almost every orbit is convergent, i.e. asymptotic to some equilibrium point. However, some application problems do generate dynamical systems whose equilibrium point sets have accumulation points, and therefore quasiconvergence does not imply convergence. Simple examples are the following functional differential equation

$$
\dot{x}(t)=-f(x(t))+f(x(t-r))
$$

describing a compartmental system with one pipe, the motion of a classically radiating electron, epidemics and population growth, where $r>0, f: R \rightarrow R$ is continuous and increasing; or the following partial differential equation

$$
\begin{aligned}
\frac{\partial u}{\partial t} & =A u+g(x, u, \nabla u), \quad t>0, \quad x \in \Omega \\
u(x, 0) & =v(x), \quad x \in \bar{\Omega}, \\
\frac{\partial u(x, t)}{\partial n} & =0, \quad x \in \partial \Omega, t \geq 0
\end{aligned}
$$

where $\Omega$ is a bounded smooth domain in $R^{n}, A$ is a second-order uniformly elliptic differential operator and $g: \Omega \times R \times R^{n} \rightarrow R$ is locally Lipschitz and satisfies $g(x, c, 0)=0$ for any $x \in \Omega$ and $c \in R, \partial u / \partial n$ is the derivative of $u$ in the direction of the outward normal to $\Omega$. For these equations, each

Received by the editors October 3, 1988 and, in revised form December 2, 1988.

1980 Mathematics Subject Classification (1985 Revision). Primary 06F05, 34K20, 34K25, $34 \mathrm{C} 35,35 \mathrm{~K} 10,58 \mathrm{~F} 10,92 \mathrm{~A} 15$. 
constant function is a solution, the set of equilibria has accumulation points, and thus quasiconvergence can tell us nothing about asymptotic behaviors of solutions but boundedness.

In this paper, we announce some convergence criteria for strongly monotone dynamical systems whose set of equilibrium points possesses a minimal or stable property. We will also present some examples to show how to apply our results to functional differential equations and partial differential equations which have infinitely many equilibrium points.

Let $X$ be a Banach lattice such that $(X, \leq,\|\cdot\|)$ has order-continuous norm. A dynamical system $\Phi=\left\{\phi_{t}\right\}_{t \geq 0}$ on $X$ is monotone, if $y>x$ and $t \geq 0$ imply $\phi_{t}(y) \geq \phi_{t}(x)$. A monotone dynamical system $\Phi=\left\{\phi_{t}\right\}_{t \geq 0}$ on $X$ is eventually strongly monotone if there exists a constant $T>0$ such that $y>x$ and $t \geq T$ imply $\phi_{t}(y) \gg \phi_{t}(x)$.

Let $\gamma^{+}(x)=\left\{\phi_{t}(x) ; t \geq 0\right\}$ denote the positive semiorbit of $x, \omega(x)$ be the $\omega$-limit set and $E$ be the set of all equilibrium points, that is, $E=\{x \in$ $X ; \phi_{t}(x)=x$ for all $\left.t \geq 0\right\}$. A point $x \in X$ (or equivalently, the orbit $\gamma^{+}(x)$ ) is convergent if $\lim _{t \rightarrow \infty} \phi(t, x)$ exists and the limit is in $E$. The following minimal property is essential throughout this paper.

Definition 1. A closed subset $M \subseteq E$ possesses minimal property with respect to a positive invariant subset $U$ if, there exists a continuous function $e: U \rightarrow$ $M \cap U$ so that

(1) $x-e(x) \in X_{+}-$Int $X_{+}$for any $x \in U$,

(2) if $y \in M$ and $y \ll x$, then $y \leq e(x)$.

Theorem 1 (Convergence criterion with minimal property). Suppose that $\left\{\phi_{t}\right\}_{t \geq 0}$ is an eventually strongly monotone dynamical system, a closed subset $M$ of $E$ possesses minimal property with respect to $U$. Then any precompact orbit $\gamma^{+}(x)$ through $x \in U$ is convergent, and $\lim _{t \rightarrow \infty} \phi(t, x) \in M$.

Sketch of Proof. Let a positive constant $T$ be given so that $\phi(t, y) \gg \phi(t, z)$ for any $y, z \in U$ with $y>z$ and for all $t \geq T$. Let $e_{i}=e(\phi(i T, x))$. Then $e_{i} \leq \phi(i T, x)$. If $e_{i} \neq \phi(i T, x)$, then

$$
e_{i}=\phi\left(T, e_{i}\right) \ll \phi(T, \phi(i T, x))=\phi((i+1) T, x)
$$

and thus $e_{i} \leq e_{i+1}$. Therefore by induction we get the following increasing sequence

$$
e_{0} \leq e_{1} \leq e_{2} \leq e_{3} \leq \cdots
$$

Therefore $\lim _{k \rightarrow \infty} e_{k}=\hat{e}$ exists by the order-continuity of the norm. Let $y \in$ $\omega(x)$. Then we can find an unbounded increasing subsequence $\left\{k_{n}\right\}_{n=1}^{\infty}$ of nonnegative integers and a sequence $\left\{\tau_{n}\right\}_{n=1}^{\infty}$ in $[0, T]$ so that $\phi\left(k_{n} T+\tau_{n}, x\right) \rightarrow y$ as $n \rightarrow \infty$. Without loss of generality we may assume that $\tau_{n} \rightarrow \tau \in[0, T]$ and $\phi\left(k_{n} T, x\right) \rightarrow z$ as $n \rightarrow \infty$. Therefore from continuity of semiflows, it follows $y=\phi(\tau, z)$. Note that $e_{k_{n}} \leq \phi\left(k_{n} T, x\right)$. By the closeness of order relation, we get $\hat{e} \leq z$ and thus $\hat{e} \leq \phi(\tau, z)=y$. That is $\hat{e} \leq \omega(x)$. By invariance of $\omega(x)$, 
we can find an element $w \in \omega(x)$ so that $\phi(\tau+T, w)=z$. If $w \neq \hat{e}$, then by eventually strong monotonicity we have $\hat{\boldsymbol{e}}=\phi(\tau+T, \hat{e}) \ll \phi(\tau+T, w)=z$. That is $z-\hat{e} \in \operatorname{Int} X_{+}$which implies that $\phi\left(k_{n} T, x\right)-e\left(\phi\left(k_{n} T, x\right)\right) \in \operatorname{Int} X_{+}$ for sufficiently large $n$, a contradiction to our assumption. Therefore $w=\hat{e}$, and thus $z=\phi(\tau+T, \hat{e})=\hat{e}$. Hence $y=\phi(\tau, \hat{e})=\hat{e}$. This shows $\omega(x)=$ $\{\hat{e}\} \subseteq M \subseteq E$.

A simple example is the following two-dimensional compartmental system with pipes

$$
\begin{aligned}
& \frac{d}{d t} x_{1}(t)=-g_{11}\left(x_{1}(t)\right)-g_{21}\left(x_{1}(t)\right)+g_{11}\left(x_{1}\left(t-r_{11}\right)\right)+g_{12}\left(x_{2}\left(t-r_{12}\right)\right) \\
& \frac{d}{d t} x_{2}(t)=g_{21}\left(x_{1}\left(t-r_{21}\right)\right)+g_{22}\left(x_{2}\left(t-r_{22}\right)\right)-g_{12}\left(x_{2}(t)\right)-g_{22}\left(x_{2}(t)\right)
\end{aligned}
$$

where $g_{i j}: R \rightarrow R$ are Lipschitz continuous and increasing with $g_{i j}(0)=0$, and $\lim _{|s| \rightarrow \infty}\left|g_{i j}(s)\right|=\infty, r_{i j} \geq 0$ are constants for $i, j=1,2$. By emplying standard comparison technique and adopting a similar argument to that in Gyori [1] and Smith [5], we can prove the solution flow defined by equation (3) defines an eventually strongly monotone semiflow on $\left.P=C\left(\left[-r_{1}, 0\right], 0, \infty\right)\right) \times$ $C\left(\left[-r_{2}, 0\right],[0, \infty)\right)$ where $r_{i}=\max \left\{r_{1 i}, r_{2 i}\right\}, i=1,2$. It is clear that the set $E$ of equilibrium points contains the following set of constant functions

$$
M=\left\{\left(\hat{c}_{1}, \hat{c}_{2}\right) ;\left(c_{1}, c_{2}\right) \in R \times R \text { and } g_{21}\left(c_{1}\right)=g_{12}\left(c_{2}\right)\right\}
$$

where $\hat{c}_{i}$ is a constant function on $\left[-r_{i}, 0\right]$ with the value $c_{i} \in R$. By constructing a function $e: P \rightarrow M \subseteq E$ as following

$$
e\left(\phi_{1}, \phi_{2}\right)=\left\{\begin{array}{l}
\left(g_{21}^{-1} g_{12}\left(\min _{s \in\left[-\hat{r}_{2}, 0\right]} \phi_{2}(s)\right), \min _{s \in\left[-\hat{r}_{2}, 0\right]} \phi_{2}(s)\right. \\
\quad \text { if } \min _{s \in\left[-r_{1}, 0\right]} \phi_{1}(s) \geq g_{21}^{-1} g_{12}\left(\min _{s \in\left[-r_{2}, 0\right]} \phi_{2}(s)\right) \\
\left(\min _{s \in\left[-\hat{r}_{1}, 0\right]} \phi_{1}(s), g_{12}^{-1} g_{21}\left(\min _{s \in\left[-r_{1}, 0\right]} \phi_{1}(s)\right)\right) \\
\quad \text { if } \min _{s \in\left[-r_{1}, 0\right]} \phi_{1}(s) \leq g_{21}^{-1} g_{12}\left(\min _{s \in\left[-r_{2}, 0\right]} \phi_{2}(s)\right) .
\end{array}\right.
$$

We can verify that the set $M$ possesses minimal property. Therefore by Theorem 1 , any orbit $\gamma^{+}\left(\phi_{1}, \phi_{2}\right)$ through $\left(\phi_{1}, \phi_{2}\right) \in P$ is convergent.

As an immediate consequence of Theorem 1, we get the following sufficient condition for asympotic constancy:

Corollary (Asymptotic constancy criterion). Suppose I is a compact topological space or a smooth compact n-dimensionoal submanifold of $R^{n}, C^{r}(I)$ denotes the Banach lattice of $C^{r}$ maps $U: I \rightarrow R^{k}$ such that $U$ and its derivatives of order $\leq r$ are continuous, endowed with the usual $C^{r}$ norm and the usual functional ordering. Suppose $\left\{\phi_{t}\right\}_{t \geq 0}$ is an eventually strongly monotone dynamical system on $C^{r}(I)$ and the set of equilibrium points contains all constant functions. Then each precompact orbit converges to a constant function as $t \rightarrow \infty$. 
Sketch of Proof. Let

$$
M=\{\hat{c} ; c \in R \text { and } \hat{c} \text { denotes the constant function on } I \text { with the value } c\} \text {. }
$$

By assumption $M \subseteq E$, the set of equilibria. Evidently, $M$ possesses the minimal property with respect to $C^{r}(I)$ since the map $e: C^{r}(I) \rightarrow M$ defined

$$
e(u)=\min _{x \in I} u(x)
$$

satisfies the conditions in Definition 1. Therefore our conclusion follows trivially from Theorem 1 .

According to the above criterion, each solution of the functional differential equation (1) or partial differential equation (2) is convergent.

The following theorem, based on the general quasiconvergence criterion by Hirsch [3], provides another sufficient condition for convergence.

Theorem 2 (Convergence criterion with stable equilibria). Suppose that $\left\{\phi_{t}\right\}_{t \geq 0}$ is an eventually strongly monotone dynamical system such that each orbit is precompact and upper stable, and that any equilibrium point is stable. Then any orbit is convergent.

Here and hereafter, an equilibrium point $x \in X$ is stable if, for any given $\varepsilon$ there exists $\delta=\delta(\varepsilon, x)>0$ such that $\gamma^{+}(y) \subseteq U_{\varepsilon}(x)$ for any $y \in U_{\delta}(x)$, where $U_{\varepsilon}$ is the $\varepsilon$-neighborhood. An orbit $\gamma^{+}(x)$ is upper stable if for every $\varepsilon>0$ there exists $\delta>0$ such that if $y>x$ and $\hat{d}(y, x) \leq \delta$, then $\hat{d}(\phi(t, y), \phi(t, x))<\varepsilon$, where $\hat{d}$ is a metric defining the order topology of $X$.

Sketch of Proof. Let $\gamma^{+}(x)$ be a fixed orbit. Employing the argument of [3, Theorem 8.3] we can prove that $\gamma^{+}(x)$ is convergent to $\omega(x) \subseteq E$. Therefore our conclusion follows trivially from the stability of each equilibrium point.

As an illustrating example, we consider an $n$-compartmental system with pipes described by the following functional differential equation

$$
\frac{d}{d t} x_{i}(t)=-\sum_{j=1}^{n} g_{j i}\left(x_{i}(t)\right)+\sum_{j=1}^{n} g_{i j}\left(x_{j}\left(t-r_{i j}\right)\right), \quad i=1, \ldots, n
$$

where $g_{i j}(u)$ is monotone increasing, continuous and locally Lipschitz with $g_{i j}(0)=0$. The phase space is chosen as $C=C\left(\left[-r_{1}, 0\right],[0, \infty)\right) \times \cdots \times$ $C\left(\left[-r_{n}, 0\right],[0, \infty)\right)$ where $r_{i}=\max _{j=1, \ldots, n} r_{j i}$. It has been proved that for any $\left(\phi_{1}, \ldots, \phi_{n}\right) \in C$ there is a unique non-negative solution on $[0, \infty)$ which satisfies the following law of energy

$$
\sum_{i=1}^{n}\left[x_{i}(t)+\sum_{j=1}^{n} \int_{t-r_{i j}}^{t} g_{i j}\left(x_{j}(s)\right) d s\right]=\text { const }
$$

for all $t \geq 0$. Moreover, in $\mathrm{Wu}$ [6], we proved that the semiflow defined by the above equation is eventually strongly monotone and the law of energy implies the upper stability of any non-negative orbit, the stability of equilibrium points and precompactness of any orbit in $C$. Therefore as an immediate consequence 
of Theorem 2, we can assert that any orbit of compartmental systems with pipes is convergent.

\section{REFERENCES}

1. I. Gyori, Connections between compartmental systems with pipes and integrodifferential equations, Math. Modelling 7 (1986), 1215-1238.

2. P. Hess, On stabilization of discrete strongly order-preserving semigroups and dynamical processes, Proceedings of Trends in Semigroup Theory and Applications, Trieste, September 28-October 2, 1987.

3. M. Hirsch, Stability and convergence in strongly monotone dynamical systems, J. Reine Angew Math. 383 (1988), 1-53.

4. X. Mora, Semilinear problems define semiflows on $C^{k}$ spaces, Trans. Amer. Math. Soc. 278 (1983), 1-55.

5. H. Smith, Monotone semiflows generated by functional differential equations, J. Differential Equations 66 (3) (1987), 420-442.

6. Jianhong $\mathrm{Wu}$, Monotone semiflows on product spaces and active compartmental systems with pipes (submitted).

Department of Mathematical Sciences, Memphis State University, Memphis, TENNESSEE 38152

Current address: Department of Mathematics, University of Alberta, Edmonton, Alberta, Canada T6G 2G1 